\title{
A PVT insensitive boosted charge transfer for high speed charge-domain pipelined ADCs
}

\author{
Zhenhai Chen $^{1,2 a)}$, Zongguang $\mathbf{Y u}^{1,2}$, Songren Huang ${ }^{1,2}$, \\ Hong Zhang ${ }^{3}$, and Huicai $\mathbf{J i}^{2}$ \\ ${ }^{1}$ Wide Bandgap Semiconductor Technology Disciplines State Key Laboratory, School \\ of Microelectronics, Xidian University, Xi'an, 710071, China \\ ${ }^{2}$ China Electronic Technology Group Corporation, No.58 Research Institute, Wuxi \\ 214035, China \\ ${ }^{3}$ School of Electronics and Information Engineering, Xi'an Jiaotong University, \\ Xi'an, 710049, China \\ a)chenzh@mail.xidian.edu.cn
}

\begin{abstract}
A process, voltage, temperature (PVT) insensitive boosted charge transfer (BCT) circuit for charge-domain (CD) pipelined analog-to-digital converters (ADC) is presented. The output charge of existing BCT varies extensively with PVT variation, leading to large common-mode charge errors in each differential BCT stage when used in CD pipelined ADCs. Therefore, complicate commonmode control circuits must be adopted to stabilize the common-mode charge of each stage, which consumes large power and chip area. The proposed BCT circuit employs a differential difference amplifier and a differential voltage reference to reject the charge errors caused by PVT variations. A 125-MSPS, 10-bit CD pipelined ADC without commonmode control circuit is implemented based on the proposed BCT, consuming only $27 \mathrm{~mW}$ from a $1.8 \mathrm{~V}$ supply.
\end{abstract}

Keywords: charge domain, pipelined ADC, charge transfer circuit

Classification: Integrated circuits

\section{References}

[1] M. Anthony, E. Kohler, J. Kurtze, L. Kushner, and G. Sollner, "A processscalable low-power charge-domain 13-bit pipeline ADC," IEEE Symp. VLSI Circuits, pp. 222-223, June 2008.

[2] M. Anthony and G. Sollner, "Ultra-low-power 500-MSPS 12-bit A/D converter using interleaving and CMOS charge-domain technology," Annual Compound Semiconductor Integrated Circuit Symposium, pp. 1-4, Oct. 2009.

[3] M. Yoshioka, M. Kudo, K. Gotoh, and Y. Watanabe, "A 10 b 125 MS/s $40 \mathrm{~mW}$ pipelined ADC in $0.18 \mu \mathrm{m}$ CMOS," ISSCC Dig. Tech. Papers, pp. 282-283, 2005.

[4] K.-H. Lee, S.-W. Lee, and Y.-J. Kim, "Ten-bit $100 \mathrm{MS} / \mathrm{s} 24.2 \mathrm{~mW} 0.8 \mathrm{~mm}^{2}$ $0.18 \mu \mathrm{m}$ CMOS pipeline ADC based on maximal circuit sharing schemes," 
IET Electronics Letters, vol. 45, no. 25, pp. 588-589, Dec. 2009.

[5] J. Li and U. K. Moon, "A 1.8-V 67-mW 10-bit 100-MS/s pipelined ADC using time-shifted CDS technique," IEEE J. Solid-State Circuits, vol. 39, no. 9 , pp. $1468-1476$, Sept. 2004.

\section{Introduction}

The charge domain (CD) pipelined ADC based on boosted charge transfer $(\mathrm{BCT})$ circuits is a newly emerging opamp-less ADC architecture [1, 2], which can achieve high speed A/D conversion at lower power dissipation compared with those conventional pipelined ADCs based on switched-capacitor architectures $[3,4,5]$. However, the output charge amount of the BCT in $[1,2]$ is influenced remarkably by process, voltage and temperature (PVT) variations, which can cause large fluctuations in the common-mode charge when the BCTs are used in CD pipelined ADCs. The common-mode charge errors reduce the input signal range of the ADC extensively. Furthermore, as common-mode charge error accumulates stage by stage, several stages at the backend of the pipeline may fail to function when common-mode charge error increases to a given extent. In order to solve the problem, complicated common-mode charge control techniques are introduced in $[1,2]$ to stabilize common-mode charge in each stage, which increases the design complexity, chip area and power consumption. This letter presents a new BCT that adopts a differential difference amplifier (DDA) with a differential voltage reference to reject the output common-mode charge errors caused by PVT variations. With the proposed $\mathrm{BCT}$, common-mode charge control circuits can be eliminated in $\mathrm{CD}$ pipelined ADCs, and even lower chip area and power consumption can be achieved.

\section{BCT circuits}

\subsection{Analysis of the existing BCT}

The BCT circuit in [1] and its operating waveforms are given in Fig. 1 (a). The gate of $M_{S}$ is controlled by the output of amplifier $A_{1}$, which is composed of $\mathrm{M}_{1}, \mathrm{M}_{2}$ and $\mathrm{M}_{3}$. Before $t_{1}, \Phi_{1}$ is in high state, and $\mathrm{M}_{\mathrm{S}}$ is initially turned off. The source node (S) and the drain node (D) of $\mathrm{M}_{\mathrm{S}}$ are set to reference voltages $V_{p 1}$ and $V_{p 2}$, respectively. The input signal $V_{i n}$ is tracked and converted into charge by capacitor $C_{1}$. The charge transferring process is initiated at $t_{1}$ when $\Phi_{1}$ changes to low state and the voltage at node $\mathrm{A}, V_{\mathrm{A}}$, falls to ground through switch $\mathrm{S}_{2}$. The voltage change of $V_{A}$ causes a voltage drop at node $\mathrm{S}\left(V_{\mathrm{S}}\right)$, which in turn induces a larger increase in $V_{\mathrm{G}}$ because of amplifier $\mathrm{A}_{1}$. The large voltage difference between $V_{\mathrm{G}}$ and $V_{\mathrm{S}}$ turns on $\mathrm{M}_{\mathrm{S}}$. Due to the difference between $V_{D}$ and $V_{S}$, charge stored on $C_{1}$ transfers onto $C_{2}$, which causes $V_{S}$ to rise and $V_{D}$ to fall. Then, amplifier $\mathrm{A}_{1}$ drives $\mathrm{V}_{\mathrm{G}}$ to fall gradually. At time $t_{2}$, when $V_{S}$ settles to the cut-off value $V_{0}, \mathrm{M}_{\mathrm{S}}$ is turned off and the charge transfer process is terminated. At $t_{3}$, the next 
charge conversion and transferring process is started. The charge transferred in the whole process can be expressed in terms of the voltage change across capacitor $C_{1}$,

$$
\begin{aligned}
Q_{T} & =C_{1} \cdot\left\{\left[V_{A}\left(t_{2}\right)-V_{A}\left(t_{1}\right)\right]-\left[V_{S}\left(t_{2}\right)-V_{S}\left(t_{1}\right)\right]\right\} \\
& =C_{1} \cdot\left[-V_{i n}\left(t_{1}\right)+V_{P 1}-V_{0}\right],
\end{aligned}
$$

where $V_{0}$ is the voltage at node $\mathrm{S}$ when $\mathrm{M}_{\mathrm{S}}$ is just turned off, which is determined both by the size and threshold voltage of $\mathrm{M}_{\mathrm{S}}$, and the input/output characteristics of amplifier $\mathrm{A}_{1}$. Under ideal PVT conditions, $V_{0}$ is almost a constant [1].

In CD pipelined ADCs, two BCTs are employed in a conversion stage to perform differential signal processing [1]. Assuming the input signals of the differential stage are $V_{i n p}$ and $V_{i n n}$, the differential charge, $Q_{T, \text { diff }}$, and the common-mode charge, $Q_{T, C M}$, can be obtained from Eqn. (1):

$$
\begin{aligned}
& Q_{T, \text { diff }}=C_{1} \cdot\left[V_{i n p}\left(t_{1}\right)-V_{i n n}\left(t_{1}\right)\right] \\
& Q_{T, C M}=C_{1} \cdot\left[-V_{i n, C M}+V_{P 1} / 2-V_{0} / 2\right]
\end{aligned}
$$

where $V_{i n, C M}=\left(V_{i n p}+V_{i n n}\right) / 2$, is the common-mode level of the input differential voltage. From Eqn. (2), the differential output charge is a linear function of the differential input voltage at $t_{1}$. Therefore, A/D conversion can be performed by quantization of the differential voltage at node $\mathrm{D}$ between $t_{1}$ and $t_{2}$. Conditional charge subtracting (or adding) circuits are also needed at node $\mathrm{D}$ to form a complete $\mathrm{CD}$ pipeline stage [1], which are omitted in Fig. 1 for simplicity.

As shown in Eqn. (1), the common-mode charge is determined mainly by $V_{i n, C M}, V_{P 1}$, and $V_{0}$. The input common-mode level, $V_{i n, C M}$, can be set by off-chip common-mode circuit, and $V_{P 1}$ is often generated by a bandgap reference. Therefore, $V_{i n, C M}$ and $V_{P 1}$ is relatively stable under PVT variations. However, because $V_{0}$ is mainly determined by the DC operating point of amplifier, $\mathrm{A}_{1}$, it will vary extensively with PVT and cause large common-mode charge errors.

\subsection{The proposed PVT insensitive BCT}

The proposed BCT structure and its operating waveforms is given in Fig. 1(b), in which a DDA is used to detect the voltage difference between node $\mathrm{D}$ and node $\mathrm{S},\left(V_{\mathrm{D}}-V_{\mathrm{S}}\right)$, and compare it with a differential reference $V_{r 2^{-}}$ $V_{r 1}$. The charge transferred can be expressed in term of the voltage change across $C_{1}$ or $C_{2}$ :

$$
Q_{T}=C_{1} \cdot\left\{\left[V_{A}\left(t_{2}\right)-V_{A}\left(t_{1}\right)\right]-\left[V_{S}\left(t_{2}\right)-V_{S}\left(t_{1}\right)\right]\right\}=C_{2}\left[V_{D}\left(t_{2}\right)-V_{D}\left(t_{1}\right)\right]
$$

After $t_{2}$, the DDA set $V_{D}-V_{S}$ towards $V_{r 2}-V_{r 1}$ :

$$
V_{D}\left(t_{2}\right)-V_{S}\left(t_{2}\right)=V_{r 2}-V_{r 1}
$$

Applying Eqn. (4) into Eqn. (3) and substituting each voltage term with its corresponding value, the transferred charge can be recalculated as

$$
Q_{T}=\left[-V_{i n}\left(t_{1}\right)+\left(V_{p 2}-V_{p 1}\right)-\left(V_{r 2}-V_{r 1}\right)\right] \cdot\left[C_{1} C_{2} /\left(C_{1}+C_{2}\right)\right]
$$



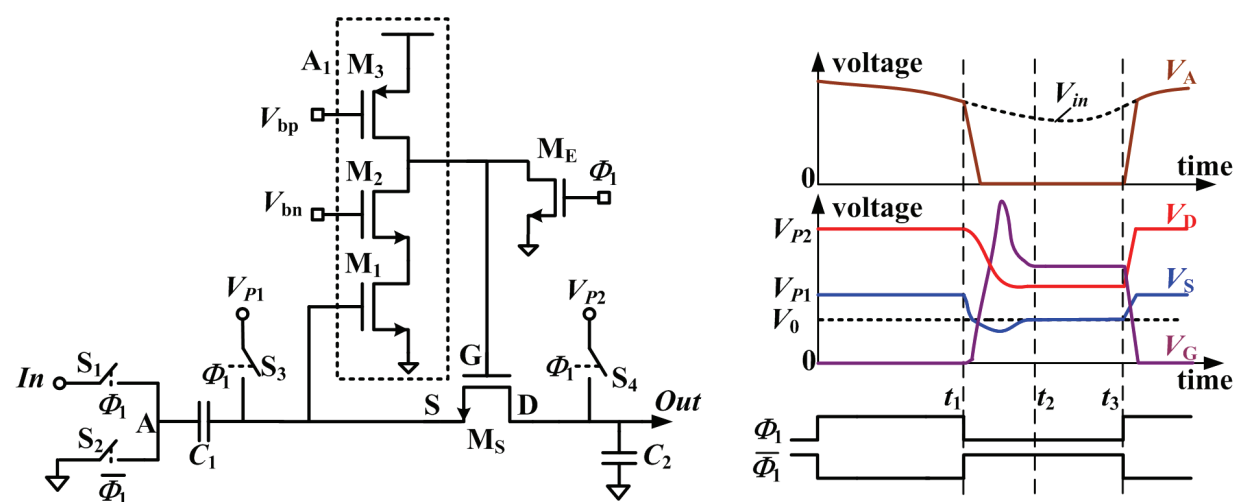

(a)
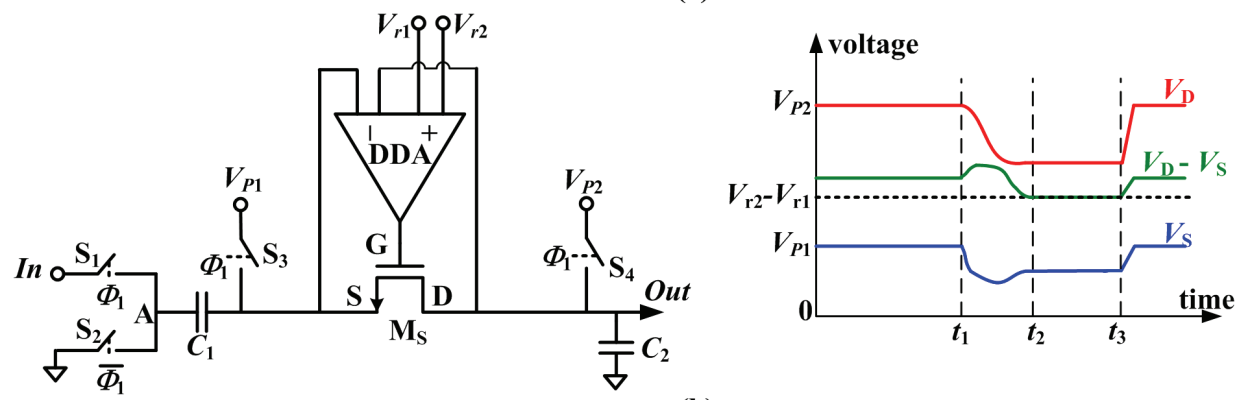

(b)
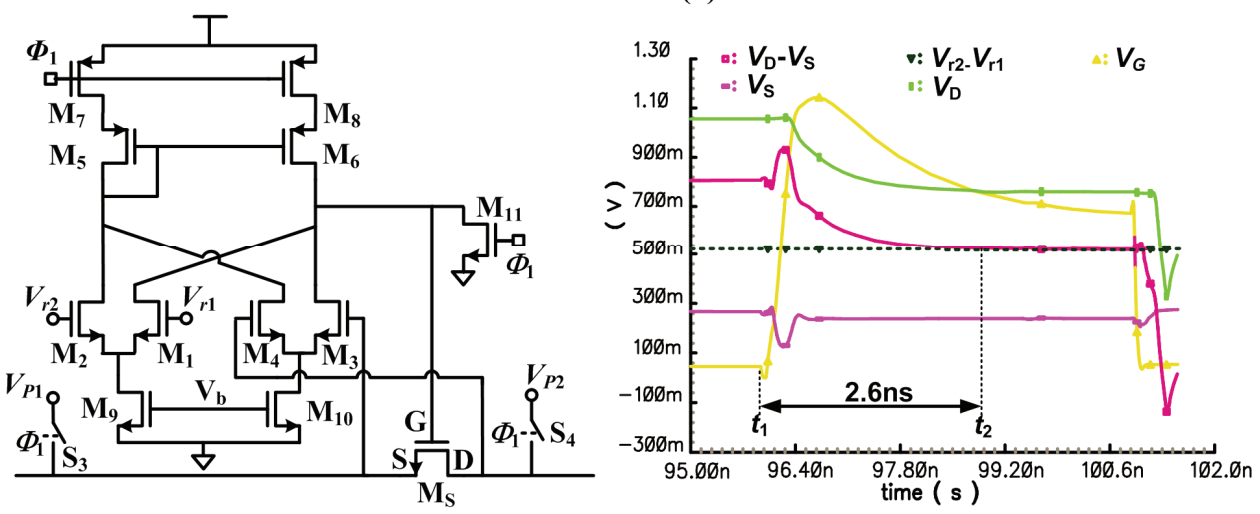

(c)

Fig. 1. BCTs and operation waveforms: (a) the BCT in $[2]$; (b) the proposed BCT; (c) the schematic and simulated waveform of the proposed BCT.

For a differential conversion stage composed of two proposed BCTs, the differential and common-mode charge can be expressed as

$$
\begin{aligned}
& Q_{T, \text { diff }}=\left[V_{i n p}\left(t_{1}\right)-V_{i n n}\left(t_{1}\right)\right] \cdot\left[C_{1} C_{2} /\left(C_{1}+C_{2}\right)\right] \\
& Q_{T, C M}=\left[-V_{i n, C M}+\left(V_{p 1}-V_{p 2}\right) / 2-\left(V_{r 2}-V_{r 1}\right) / 2\right] \cdot\left[C_{1} C_{2} /\left(C_{1}+C_{2}\right)\right]
\end{aligned}
$$

From Eq. (6), $Q_{T, \text { diff }}$ is still a linear function of the differential input voltage at $t_{1}$. However, comparing to Eq. (2), $Q_{T, C M}$ of the proposed circuits is mainly determined by $V_{i n, C M}$ and the difference of two pair of reference voltages. Hence, it is more insensitive to PVT variations than that of $\mathrm{BCT}$ in Fig. 1 (a).

Circuit implementation of the proposed BCT is shown in Fig. 1(c), in which the input and output switch capacitor circuits are omitted for simplicity. Simulated transient waveforms of the proposed circuit with a clock 
frequency of $125 \mathrm{MHz}$ are given in Fig. 1 (c). The charge transferring process is initiated at $t_{1}$ as discussed above. The DDA senses the large value of $V_{D^{-}} V_{S}$ and turns on $\mathrm{M}_{\mathrm{S}}$. The value of $V_{D^{-}} V_{S}$ reduces gradually and settles to $V_{r 2}-V_{r 1}$ at $\mathrm{t}_{2}$ when $V_{\mathrm{G}}$ falls to a given low value and turns off $\mathrm{M}_{\mathrm{S}}$. The simulated settling time is about $2.6 \mathrm{~ns}$, which means the proposed BCT can work under a clock frequency above $125 \mathrm{MHz}$.

\section{Simulation and measurement results}

The proposed BCT is designed with TSMC $0.18 \mu \mathrm{m}$ CMOS technology, and simulated at a $1.8 \mathrm{~V}$ power supply. A sample circuit for the BCT in Fig. 1 (a) is also designed for comparison. The capacitors and the transistor $\mathrm{M}_{\mathrm{S}}$ of both BCTs are designed with the same sizes $\left(C_{1}=2 * C_{2}=1 \mathrm{pF},(W / \mathrm{L})_{\mathrm{MS}}=\right.$ $20 \mu / 0.3 \mu$ ). The amplifier $A_{1}$ in Fig. 1 (a) and the DDA in Fig. 1 (c) are both designed with a gain of $30 \mathrm{~dB}$. An equivalent charge expression is chosen for both BCTs to compare charge waveforms:

$$
Q_{e}=\left(V_{D}-V_{S}\right) \cdot C_{1} C_{2} /\left(C_{1}+C_{2}\right)
$$
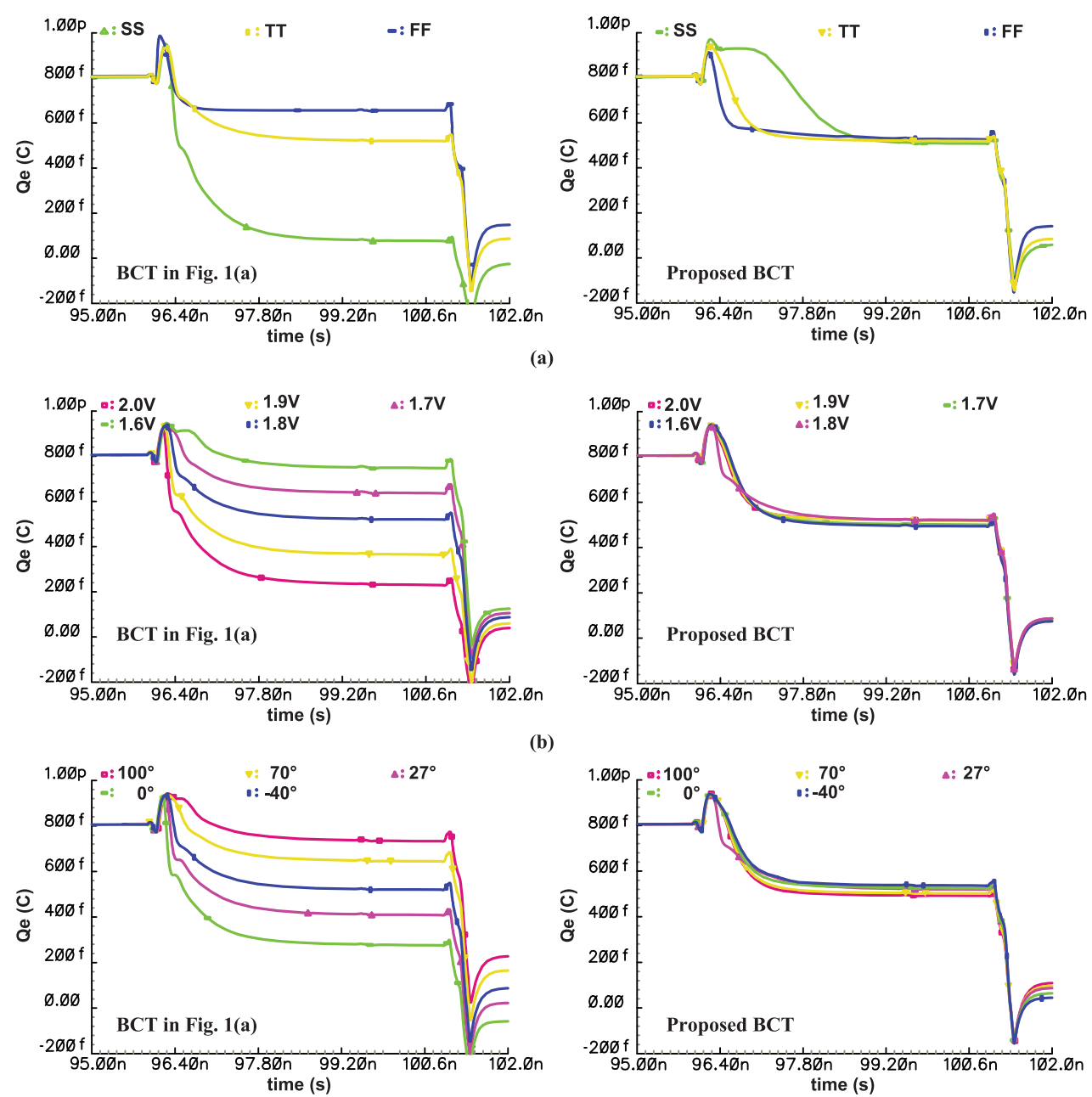

)

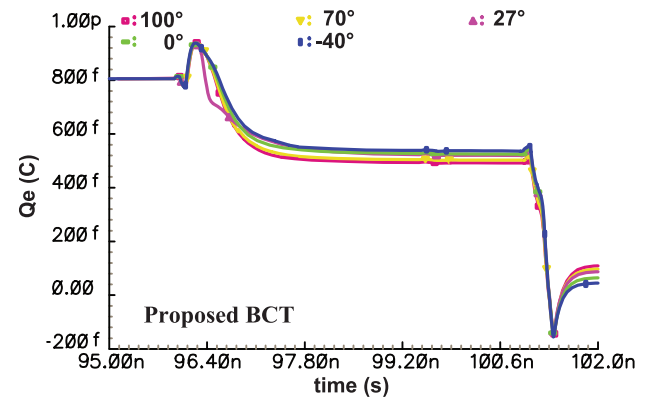

(c)

Fig. 2. Comparisons of simulation results under different conditions: (a) process corners; (b) supply voltages; (c) temperature. 
The simulated $Q_{e}$ under different process corners (SS for slow, TT for typical and FF for fast), temperatures, and supply voltages are given in Fig. 2.

As can be seen from Fig. 2, the charge waveforms of the BCT in Fig. 1 (a) vary significantly with large PVT variations, while the waveforms of proposed BCT keep almost unchanged, which verifies that the proposed BCT can reject charge errors due to PVT variations remarkably. The proposed BCT's power consumption is about twice of the conventional one. However, this power consumption increase is much smaller than the power consumption of common mode control circuit used in the conventional CD pipelined ADCs.

A 10-bit 125-MSPS CD pipelined ADC is designed and fabricated based on the proposed $\mathrm{BCT}$ without using any common mode charge control technique, with chip photo given in Fig. 3 (a). The chip area is $1.4 \times 1.0 \mathrm{~mm}^{2}$. The measured FFT spectrum, DNL and INL curves are shown in Fig. 3 (b), (c) and (d), respectively. The prototype ADC achieves a SFDR of $67.7 \mathrm{~dB}$, a SNDR of $57.3 \mathrm{~dB}$, a DNL of $0.5 \mathrm{LSB}$, and an INL of $0.7 \mathrm{LSB}$ with a $3.79-\mathrm{MHz}$ sinusoid input signal at full sampling rate, consuming only $27 \mathrm{~mW}$ from a $1.8-\mathrm{V}$ power supply.
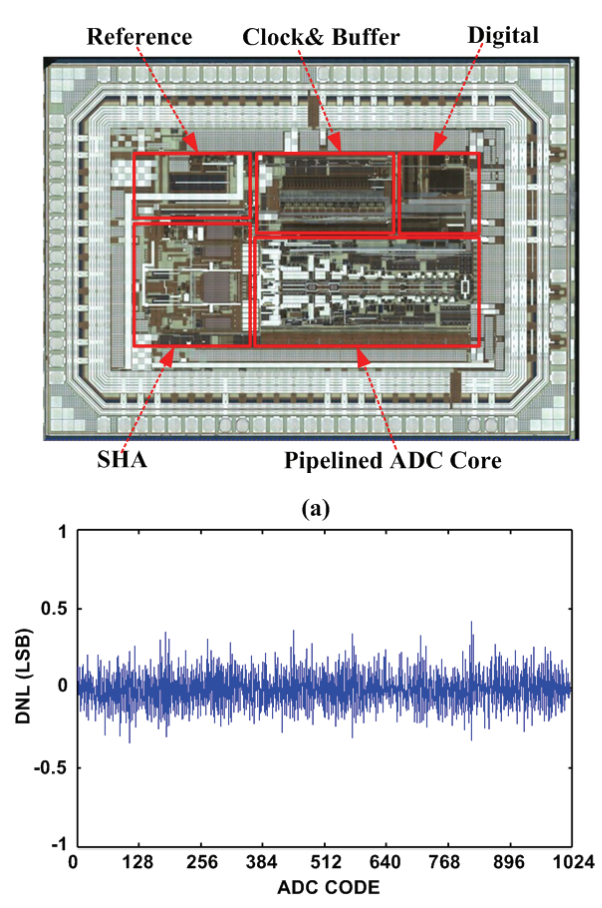

(c)

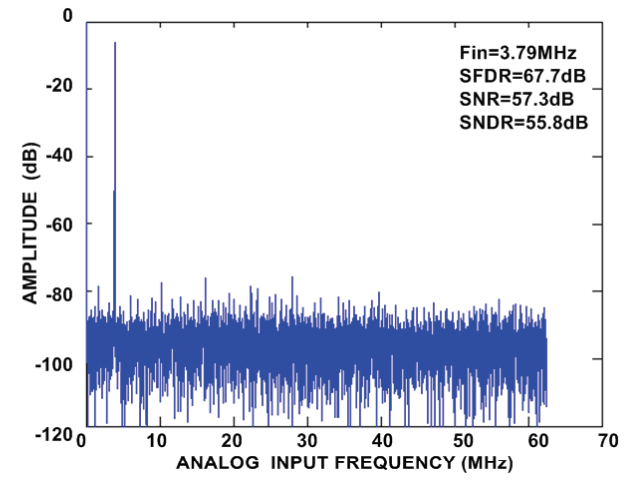

(b)

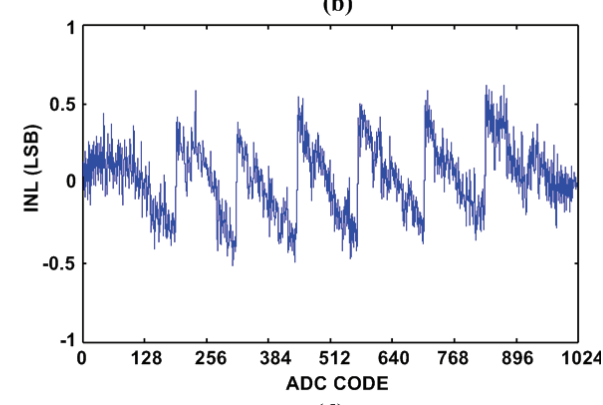

(d)

Fig. 3. Die photograph and measured results of the prototype ADC: (1) die photograph; (b) FFT spectrum; (c) DNL curve; (d) INL curve.

\section{Conclusion}

This paper presents a PVT insensitive BCT for high speed CD pipelined ADCs. Simulation results show that the PVT variation rejection of the proposed circuit is much improved compared with existing BCTs. The proposed 
BCT is verified by a $27 \mathrm{~mW} 10$-bit 125-MSPS CD pipelined ADC without using any common mode charge control techniques.

\section{Acknowledgments}

This work was supported by National Science Foundation of China under grant No.61106027. 